\title{
New CLEM Method to Reveal Ultrastructural Reorganization in the Host Cell during Coronavirus Infection
}

\author{
Gabriella Kiss ${ }^{1}$, Cedric Bouchet-Marquis ${ }^{1}$, Lee Pullan ${ }^{1}$, Doug Keene ${ }^{2}$, Benjamin W. Neuman ${ }^{3}$
}

${ }^{1}$ FEI Company, Hillsboro, OR, USA

${ }^{2}$ Shriners Hospital, Micro-Imaging Center, Portland, OR, USA

${ }^{3}$ University of Reading, UK

Correlative light and electron microscopy (CLEM) combines localization data from fluorescent microscopy (FM) with ultra-structural information from electron microscopy (EM). Recent improvements on fluorescent dyes and proteins enabled wider applications of this approach [1][2]. One of the main difficulties in CLEM is that often time, sample processing for EM imaging quenches the FM signal. Despite those difficulties, some newer labeling methods enable to perform CLEM experiments with a high level of success. The study of mouse hepatitis virus replication in murine cells described below demonstrates the most recent improvement made in this field.

Mouse hepatitis virus (MHV) belongs to the Coronavirus family. Alongside other positive-strand RNA viruses, MHV induce many subcellular changes in the host cell during infection [3]. These subcellular reorganizations mainly affect lipid structures and leads to further compartmentalization. This compartmentalization has been shown to be very important for the viral replication both functionally and strategically [4]. During this process, MHV reshapes the endoplasmic reticulum (ER) membranes into double membrane vesicles (DMVs). These DMVs contain the viral RNA to protect it from the host cell antiviral machinery. After the structural proteins are also transported to the DMVs, viral budding can occur. DMVs are thought to form a network with actively growing and maturing in the infected cell according to the stage of infection, thus 3 dimensional (3D) structural studies are necessary to fully understand their organization. Previous studies revealed many similarities among +RNA viruses which can help to better understand viral infection mechanistic [5][6][4][7][8][9][10][11]. There is still an ongoing need to find new ways to study these structural changes during infection in the host cell.

Here we demonstrate using SYBR-Gold (Life Technologies) nucleic acid stain before EM sample processing to locate the viral factories (e.g. DMVs) in the infected cells directly. We have compared two EM sample processing methods, using LR White resin without $\mathrm{OsO}_{4}$ and a Standard EM method using EPON hard resin with $1 \% \mathrm{OsO}_{4}$ to compare the effect of $\mathrm{OsO}_{4}$ on the $\mathrm{FM}$ signal. $\mathrm{OsO}_{4}$ is known to quench the FM signal, albeit necessary for ultrastructural preservation and EM contrast [12]. As expected the $\mathrm{OsO}_{4}$ weakened the FM signal in the Standard processing method, however SYBR-Gold retained enough signal for detection, even at single DMV level. Additionally the EM contrast in the samples was good to enable tilt series data collection on regions with DMVs and budded virus particles. 

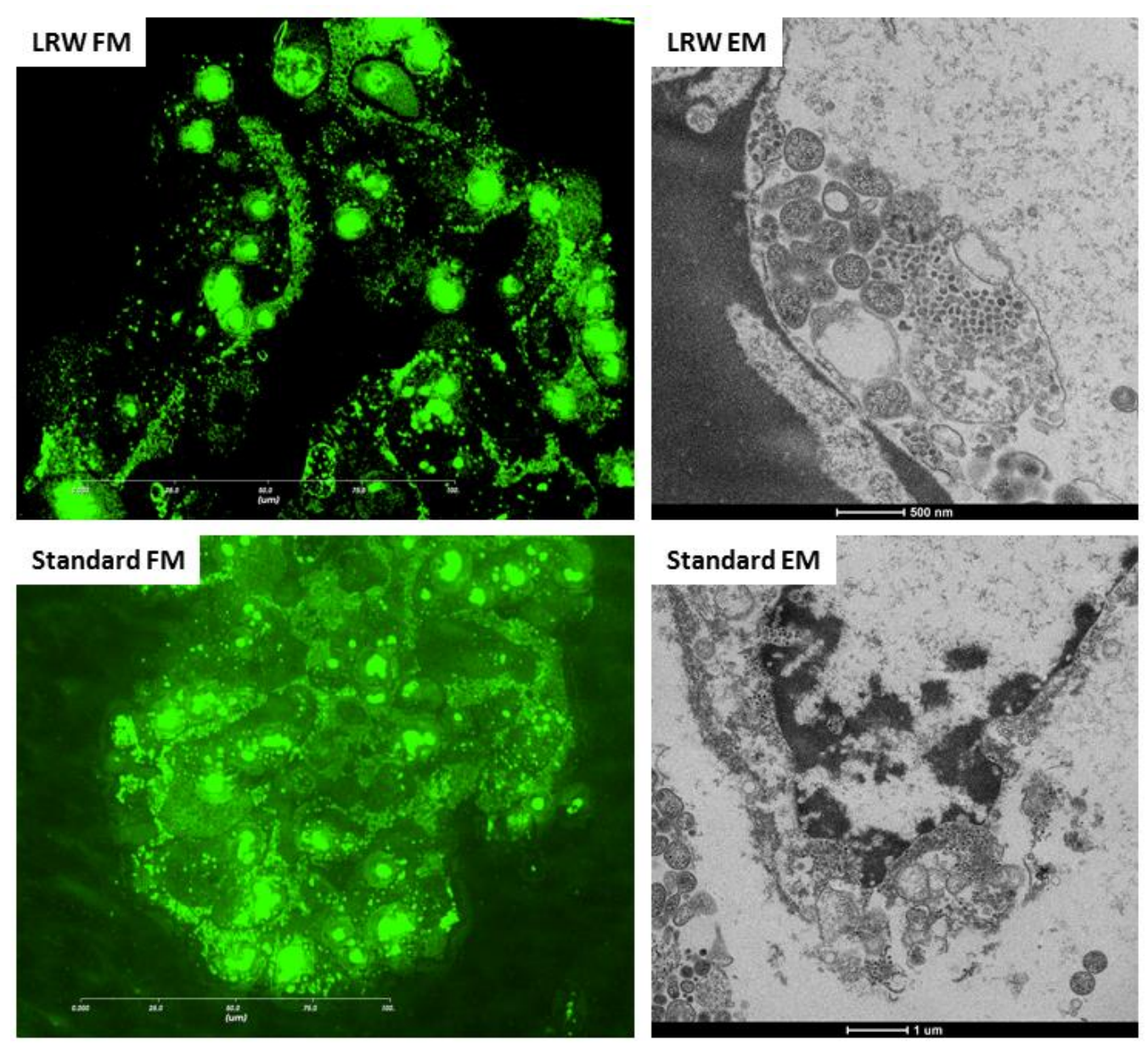

Figure 1. Fluorescent (FM) and electron microscopy (EM) images of the samples processed using LRW no $\mathrm{OsO}_{4}$ or the Standard method with $\mathrm{OsO}_{4}$ treatment. The FM signal is still visible from individual DMVs containing the viral RNA in both processing method. The quality of the EM images are also suitable for further 3D ultrastructural studies of the DMV network.

\section{$\underline{\text { References }}$}

[1] H. Xiong, et al, Nat. Commun., vol. 5, p. 3992, Jan. 2014.

[2] M. G. Paez-Segala, et al, Nat. Methods, Jan. 2015.

[3] K. Knoops, et al, J. Virol., vol. 86, no. 5, pp. 2474-87, Mar. 2012.

[4] B. G. Kopek, et al, PLoS Biol., vol. 5, no. 9, p. e220, Sep. 2007.

[5] L. K. Gillespie, et al, J. Virol., vol. 84, no. 20, pp. 10438-47, Oct. 2010.

[6] K. Knoops, et al, PLoS Biol., vol. 6, no. 9, p. e226, Sep. 2008.

[7] R. W. A. L. Limpens, et al, MBio, vol. 2, no. 5, Jan. 2011.

[8] P. Monaghan, et al, J. Gen. Virol., vol. 85, no. Pt 4, pp. 933-46, Apr. 2004.

[9] A. Schlegel, et al, J. Virol., vol. 70, no. 10, pp. 6576-88, Oct. 1996.

[10] E. J. Snijder, et al, J. Virol., vol. 80, no. 12, pp. 5927-40, Jun. 2006.

[11] S. Welsch, et al, Cell Host Microbe, vol. 5, no. 4, pp. 365-75, Apr. 2009.

[12] S. Watanabe, et al, Nat. Methods, vol. 8, no. 1, pp. 80-4, Jan. 2011. 